\title{
Oxygen ion energization by waves in the high altitude cusp and mantle
}

\author{
M. Waara ${ }^{1}$, H. Nilsson ${ }^{1}$, R. Slapak ${ }^{1}$, M. André ${ }^{2}$, and G. Stenberg ${ }^{1}$ \\ ${ }^{1}$ Swedish Institute of Space Physics, Kiruna, Sweden \\ ${ }^{2}$ Swedish Institute of Space Physics, Uppsala, Sweden \\ Correspondence to: H. Nilsson (hans.nilsson@irf.se)
}

Received: 1 June 2012 - Revised: 13 August 2012 - Accepted: 19 August 2012 - Published: 3 September 2012

\begin{abstract}
We present a comparative study of low frequency electric field spectral densities and temperatures observed by the Cluster spacecraft in the high altitude cusp/mantle region. We compare the relation between the $\mathrm{O}^{+}$temperature and wave intensity at the oxygen gyrofrequency at each measurement point and find a clear correlation. The trend of the correlation agrees with the predictions by both an asymptotic mean-particle theory and a test-particle approach. The perpendicular to parallel temperature ratio is also consistent with the predictions of the asymptotic mean-particle theory. At times the perpendicular temperature is significantly higher than predicted by the models. A simple study of the evolution of the particle distributions (conics) at these altitudes indicates that enhanced perpendicular temperatures would be observed over many $R_{\mathrm{E}}$ after heating ceases. Therefore, sporadic intense heating is the likely explanation for cases with high temperature and comparably low wave activity. We observe waves of sufficient amplitude to explain the highest observed temperatures, while the theory in general overestimates the temperature associated with the highest observed wave activity, indicating that such high wave activity is very sporadic.
\end{abstract}

Keywords. Magnetospheric physics (Magnetopause, cusp, and boundary layers; Magnetosphere-ionosphere interactions) - Space plasma physics (Wave-particle interactions)

\section{Introduction}

It is well known that ions in the ionosphere and magnetosphere can be energized in the direction perpendicular to the geomagnetic field. It is believed that transverse heating of ions is important for ion outflow, and one of the probable explanations for transverse heating is wave-particle interaction (Moore and Horwitz, 2007). One type of efficient wave energization is caused by waves near the ion gyrofrequency. This ion cyclotron resonance mechanism can be investigated using test-particle calculations or Monte Carlo simulations (Retterer et al., 1987; Chang et al., 1986).

In a recent case study, Waara et al. (2010) searched the high altitude cusp/mantle (the region of open magnetic field lines mapping to the polar cap and dominated by magnetosheath plasma) for the longest period with a significantly enhanced oxygen perpendicular to parallel temperature ratio, an expected sign of local transverse heating. They used the data set of Nilsson et al. (2006) and found a case lasting about $20 \mathrm{~min}$. It was found that the wave amplitude around the oxygen ion gyrofrequency was not high enough to explain the observed perpendicular ion temperatures using a simple ion cyclotron resonance model (Chang et al., 1986). The study of Waara et al. (2010) was followed up with a statistical study of the electric and magnetic field spectral densities in the frequency range below $1 \mathrm{~Hz}$, in the general vicinity of the high altitude oxygen gyrofrequency (Waara et al., 2011). In Waara et al. (2011), it was shown that, statistically, the gyroresonance model could, in fact, reproduce the observed average perpendicular temperature and average parallel velocity for altitudes between $8-15 R_{\mathrm{E}}$ in the cusp/mantle region given the average spectral density. It was assumed that $50 \%$ of the observed wave activity at the local $\mathrm{O}^{+}$gyrofrequency was due to left-hand polarized waves, which can effectively heat the ions. In the paper of Slapak et al. (2011), some very high temperatures could be explained using a gyroresonance model and the simultaneously observed waves. It was also shown that, for the three cases investigated, the size of the 
region of enhanced wave activity was at least one order of magnitude larger than the $\mathrm{O}^{+}$ion gyroradius.

It therefore seems as though we have some contradicting results. In our first case study, we observed a long-lasting case of strongly anisotropic ion temperatures suggesting local heating, but we could not explain the observed ion temperatures with the simultaneously observed wave activity. Choosing cases with strong wave activity (Slapak et al., 2011), we could explain the simultaneously observed ion temperatures. We also found that the average wave activity could explain the average ion temperature (Waara et al., 2011).

We follow up on these three studies with a statistical study of the correlation between ion temperature and wave intensity for each measurement point. The observations are compared with the predictions of Chang et al. (1986), both through an asymptotic mean-particle approach and a testparticle approach. These models assume continuous heating, and deviations between the model predictions and observations provide insight into the role that the sporadic nature of the heating plays.

With the approach used in our previous statistical study (Waara et al., 2011), we could not correlate the temperature and wave activity for each measurement point; we could only show that the average wave activity was suitable to explain the average ion temperature and parallel velocity. This new study therefore combines the results of the statistical study with the point-by-point comparison previously only available from the case studies. If heating is continuous, we would get as good correlation between wave activity and temperature for this point-by-point correlation study as we got for the average values. It was however shown in Waara et al. (2011) that heating, as observed by the spacecraft, is typically sporadic, lasting only a few minutes for each burst. This could strongly affect the point-by-point correlation between wave activity and ion temperature.

The correlation between the simultaneously observed ion temperature and wave activity is determined by how sporadic the waves are and how dominating the most recent heating is. The more dominating the most recent heating is, the more sporadic the ion heating can be and we will still get a good correlation. If ion heating is not sporadic, then the most recent heating needs not dominate; it is still a good measure of the integrated heating the ion has experienced along its flight path. We want to find out if we typically can explain the observed ion temperatures with the simultaneously observed waves for each measurement point. If yes, then the result of Waara et al. (2010) was clearly an exception and observed ion temperatures can in general be explained by simultaneously observed waves. If not, then the sporadic nature of the ion heating influences the statistics enough to lose a good correlation between ion temperature and wave activity. In this study, we show that there indeed is a good correlation, but also that the nature of sporadic heating causes deviation from the correlation for the strongest electric spectral densities and for the highest temperatures.

\section{Instrumentation and data}

We use data from the Cluster spacecraft (Escoubet et al., 2001). The ion data are taken from CODIF, a time-of-flight ion composition distribution function instrument that can resolve the major magnetospheric ions. The angular resolution is $22.5^{\circ}$ and the energy coverage in the modes of interest to us is from $40 \mathrm{eV}$ per charge up to $38 \mathrm{keV}$ per charge. Furthermore, we use electric field data from the electric field and wave instrument, EFW (Gustafsson et al., 2001). EFW records two orthogonal electric field components in the satellite spin plane. In the data set used in this study, the sampling rate was 25 samples/second. We also use data from the Cluster fluxgate magnetometer (FGM), which measures the magnetic field vector (Balogh et al., 2001).

The data set consists of EFW wave data when outflowing $\mathrm{O}^{+}$is seen in the energy spectrograms of the CIS CODIF data. The data set covers a 3-yr period (January to May in 2001 to 2003). This corresponds to orbits with apogee on the sunward side of the terminator plane. Only events with outflowing $\mathrm{O}^{+}$lasting more than $1 \mathrm{~h}$ were selected, and such events were seen in about two-thirds of the orbits. The particle data set is described in detail in Nilsson et al. (2006). The time series data of the electric field have been Fourier transformed to obtain frequency spectra. The record length in the Fourier transform is 1024 points. The spectral densities used in this study are averages of three partially overlapping records, shifted 512 data points with respect to each other. The DC-level $(0 \mathrm{~Hz})$ in the data is removed by subtracting the mean of each time window for the EFW. The wave data set is described in more detail by Waara et al. (2011). In that study, it was shown that the electric field to magnetic field spectral density ratio ( $E / B$ ratio) of the observed waves agreed with the Alfvén wave velocity as calculated from the background magnetic field and ion density estimates obtained using ion spectrometer data. They could also show that the altitude variation of the $E / B$ ratio in the frequency interval below $1 \mathrm{~Hz}$ was inconsistent with static current structures closing through the ionosphere. It is therefore likely that the observed wave activity at these high altitudes was due to Alfvén waves. We note that this is not always the case for lower altitudes. For example, Jasperse et al. (2006) interpreted the power law electric field frequency spectra observed by the FAST spacecraft (at an altitude of about $4000 \mathrm{~km}$ ) as being due to static structures, while peaks at the harmonics of the proton gyro frequency were interpreted as due to turbulence in the ion frame of reference. 


\section{Ion cyclotron resonance mechanism}

Chang et al. (1986) presented a theory for calculating the evolution of the perpendicular temperature in an altitudeextended, cyclotron resonant electric wave field. They also provided an asymptotic solution yielding both perpendicular and parallel temperature from the locally observed waves and the shape of the frequency spectrum. Estimates from the ion cyclotron resonance mechanism (Chang et al., 1986) give both the perpendicular and the parallel ion temperature based on the wave intensity along the ion trajectory. The heating is caused by waves near the local ion gyrofrequency along the ion trajectory. In practice, locally observed waves mapped along the magnetic field lines are used. The spectral density can often be approximated by $S(f) \propto f^{-\alpha}$, with $\alpha$ as a power law fitting parameter, and the gyrofrequency can often be assumed to fall with the cube of the geocentric distance, $f_{\mathrm{i}}(r) \propto r^{-3}$, allowing mapping to an arbitrary altitude. The mean energy ratio $W_{\perp} / W_{\|}$asymptotically approaches a constant value of $(6 \alpha+2) / 9$. In this limit, the total ion energy is insensitive to the choice of initial conditions, making it suitable for a comparison with our data. The result for the total ion energy, $W=W_{\|}+W_{\perp}$ (Retterer et al., 1987), is

$W=\left(3 \alpha+\frac{11}{2}\right)^{1 / 3} m\left[\frac{r D_{\perp}(r)}{(3 \alpha+1)}\right]^{2 / 3}$

where the quasi-linear velocity diffusion rate perpendicular to the geomagnetic field is given by

$D_{\perp}=\frac{\eta q^{2}}{4 m^{2}}\left|E_{\mathrm{x}}(\omega=\Omega)\right|^{2}$

where $q$ is the charge, $\Omega$ is the ion gyrofrequency, $\omega$ is the wave frequency, $\left|E_{\mathrm{x}}^{2}\right|$ is the electric field spectral density at the local ion gyrofrequency and $\eta$ is the proportion of the measured spectral density that corresponds to a left-hand polarized wave. We use a value of $\eta=50 \%$ in the calculations in this study. The $\alpha$ value we use is 1.5 and is taken from Waara et al. (2011).

We will compare our data with two variants of the theory of Chang et al. (1986). The first, which we call the asymptotic mean-particle theory, makes use of the equations above. In an alternative version, we release a test particle at $5 R_{\mathrm{E}}$ with average initial properties (perpendicular temperature of $17 \mathrm{eV}$, parallel velocity of $43 \mathrm{~km} \mathrm{~s}^{-1}$ (from Table 1 in Waara et al., 2011)) and follow it as it moves outward in the average observed magnetic field as function of distance. The spectral density used for each particle is the observed spectral density, using the average explicit altitude dependence of the data set as reported in Table 1 in Waara et al. (2011). The particle is followed until it reaches the altitude of observation. This calculation is performed for all our measurement points.
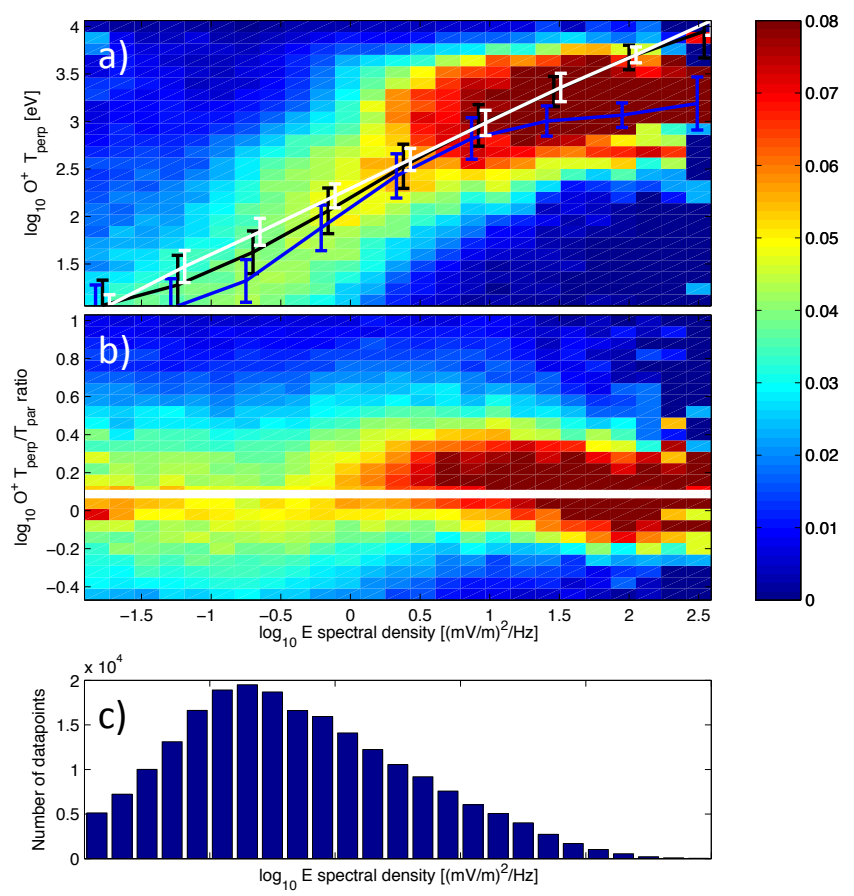

Fig. 1. (a) Distribution of $\mathrm{O}^{+}$perpendicular temperature [log $\mathrm{eV}$ ] for each interval of electric field spectral density [log $\left.\left(\mathrm{mV} \mathrm{m}^{-1}\right)^{2} \mathrm{~Hz}^{-1}\right]$. Each column is normalized; the sum of all data bins in a column is 1 . The white line is the predicted perpendicular temperature from the asymptotic mean-particle approach. The white error bars show the standard deviation for the logarithmic value. The black line is the predicted perpendicular temperature from the testparticle approach. The black error bars show the standard deviation for the logarithmic value. The blue line is the average observed perpendicular temperature. (b) Distribution of $\mathrm{O}^{+}$temperature ratio $T_{\perp} / T_{\|}$for each interval of electric field spectral density. The white line is the predicted temperature ratio from the mean-particle approach. (c) Number of data points contributing to each column.

\section{Results}

The distribution of $\mathrm{O}^{+}$perpendicular temperature for each interval of electric field spectral density is shown in panel (a) in Fig. 1. We see a strong correlation between the observed electric field spectral density and the observed perpendicular temperature.

The white line in Fig. 1a is the predicted perpendicular temperature for each electric field spectral density using the asymptotic mean-particle approach. The perpendicular temperatures are calculated for all points in the data set, and the error bars indicate the standard deviation. As can be seen, the asymptotic mean-particle theory fairly well reproduces most of the observations.

The black line in Fig. 1a is the predicted perpendicular temperature for each electric field spectral density calculated using the test-particle approach (Chang et al., 1986) for ions released with average properties from $5 R_{\mathrm{E}}$ and followed until they reach the altitude of observation, i.e. up to $15 R_{\mathrm{E}}$. 
Error bars indicate one standard deviation. This approach yields somewhat lower temperatures at low spectral density, in better but still not perfect agreement with observations. The standard deviation obtained with this method also better captures the spread of the data for low spectral densities. Otherwise there is no significant difference between the results of the two models.

Both theories predict as high temperatures as are observed for the range of electric field spectral densities. The main discrepancy between models and observations is that there is a significant number of observations of temperatures higher than predicted, especially in the spectral density range of 1$30\left(\mathrm{mV} \mathrm{m}^{-1}\right)^{2} \mathrm{~Hz}^{-1}$. For the very highest spectral densities observed, the corresponding observed temperatures are typically lower than predicted by the models. Finally, the models systematically overestimate the temperatures for the lowest spectral densities.

The blue line in Fig. 1a is the observed average perpendicular temperature. Compared to the distribution, it first seems as if the mean value is somewhat low for the highest spectral densities. However, studying the distributions bin by bin reveals a low temperature tail that is not easy to distinguish in the color distribution.

Figure 1c indicates the statistical significance of each column by showing the number of data points contributing to each column.

A higher perpendicular than parallel temperature is often a signature of local heating, as the mirror force will decrease the kinetic perpendicular temperature if the ions move up along the field line. Figure $1 \mathrm{~b}$ shows the $\mathrm{O}^{+}$perpendicular to parallel temperature ratios versus the electric field spectral density. Higher perpendicular than parallel temperature is typically observed for most of the spectral densities. The white line in Fig. 1b shows the predicted perpendicular-toparallel ratio from the asymptotic mean-particle theory. The mean energy ratio $W_{\perp} / W_{\|}$asymptotically approaches a constant value of 1.2 for the average spectral slope of our observations. As can be seen in panel (b) in Fig. 1, most of the measured values are consistent with what the asymptotic mean-particle theory predicts.

As the transversely accelerated ions subsequently move outward, their transverse energy is gradually converted to parallel energy by the mirror force. For passive adiabatic transport along the field lines, the perpendicular temperature should thus decrease. The relatively sporadic appearance of enhanced wave activity presented in Waara et al. (2011) may make it difficult to observe the actual heating, so it is not obvious that we should have a good correlation between wave activity and perpendicular temperature for each measurement point. The sporadic nature of the cusp and polar cap ion energization is also discussed by Lennartsson et al. (2004). They discussed energization of ions, which was random in both onset and location, as a plausible explanation of their data. Our results indicate that heating is indeed sporadic, but not

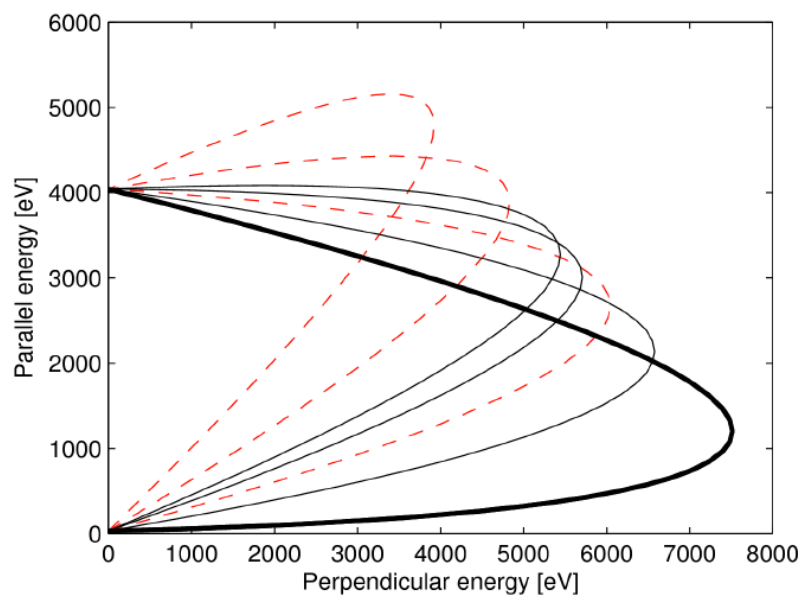

Fig. 2. Model result for an isocontour of a distribution moving to progressively higher altitude and the associated folding of the distribution function into a "conic" for a dipole field (red dashed lines) and the average observed background magnetic field (thin black lines) as function of altitude. The $\mathrm{x}$-axis shows the perpendicular energy $[\mathrm{eV}]$ and the $y$-axis the parallel energy $[\mathrm{eV}]$. The start altitude corresponds to $12 R_{\mathrm{E}}$, and the three conics correspond to the altitudes 13,14 and $15 R_{\mathrm{E}}$.

to the degree that all correlation between local wave activity and observed ion temperature is lost.

We have also studied the folding of a perpendicularly heated particle population starting at $12 R_{\mathrm{E}}$ and ending at $15 R_{\mathrm{E}}$. We let particles move outward along the field line without any heating, in order to investigate the remnant effect of hot ions after heating has ceased. The folding of a sample ion distribution is shown in Fig. 2. The initial distribution at $12 R_{\mathrm{E}}$ is seen as a thick black contour, and the thin black and red dashed contours show the evolution of the ion distribution (at 13, 14 and $15 R_{\mathrm{E}}$ ) given an observed average magnetic field and a dipole field model respectively. The decrease of the perpendicular temperature in our simple study is around $10 \%$ for each $R_{\mathrm{E}}$ if the average measured background magnetic field is used; it is around $20 \%$ for each $R_{\mathrm{E}}$ if the dipole model is used. Our results show that strongly heated ions are likely to be observed with an enhanced perpendicular temperature over a few $R_{\mathrm{E}}$ outside the actual heating region, i.e. in regions with lower electric field spectral density than predicted by the theory.

\section{Discussion}

The ion cyclotron resonance theory fairly well reproduces the observed perpendicular temperature and the temperature ratio, except for some of the highest perpendicular temperatures, where the temperatures can be up to one order of magnitude larger than predicted by the model. The ions retain their perpendicular energy some time also after leaving a heating region. One can therefore not expect a precise 
one-to-one correlation between enhanced wave activity and high perpendicular ion temperature.

The power law approximation $\left(S(f) \propto f^{-\alpha}\right)$ of the spectral density does not perfectly describe the distributions observed. The average spectral density versus frequency is dependent on altitude: the observed spectral density increases for higher altitudes. There is one order of magnitude between the highest and the lowest average spectral density over the altitude range of our data set. The electric field spectral densities (as a function of frequency) for the different altitudes generally have a power law distribution with a slope of 1.5 (Waara et al., 2011). If the explicit altitude dependence is taken into account (Chang et al., 1986), we get an effective $\alpha$ of 1.6 instead of 1.5. The small increase in $\alpha$ does not significantly change the predictions from the mean-particle theory, neither the perpendicular temperature nor the temperature ratio. Slapak et al. (2011) reported a spectral slope $\alpha$ of 2.1 for their case of strongest heating. Nor this would yield any significant increase of the perpendicular temperature for a given wave intensity as compared to the average spectral slope. The temperature ratio would increase to 1.6 , consistent with the higher temperature ratios observed for high spectral densities.

Additionally, the fact that the model assumes that the gyrofrequency falls off with the cube of the geocentric distance is not completely true for this data set. The background magnetic field presented in Waara et al. (2011) shows that a dipole model for the magnetic field is a good approximation up to $12 R_{\mathrm{E}}$, but, at higher altitudes, the decrease of the measured background magnetic field with altitude is smaller than for a dipole field. The gyrofrequency falls off on average with an exponent of 1.75 instead of with the cube of the geocentric distance (Slapak et al., 2011). The highest perpendicular temperatures are observed at the highest altitudes, where it is better to use the measured magnetic field values instead of the dipole approximation. We did this in our alternative (test particle) method, but this did not yield significantly different results as compared to the asymptotic mean-particle theory.

An explanation as to why the highest temperatures are also observed for lower spectral densities is the relatively sporadic occurrence of strongly enhanced wave activity, which makes it less likely to observe the actual heating. The heating from the waves can occur for just a few minutes, but the total energy gain for the particles remains, and the increased perpendicular temperatures remain for some time after the actual heating has stopped. Slapak et al. (2011) showed that, when picking out the highest observed wave intensities, the simultaneously observed high ion temperatures could be explained by a gyroresonance theory. It therefore seems likely that the discrepancy between the highest observed temperatures and highest simultaneously observed wave intensities is due to the temporally and/or spatially limited nature of regions of intense wave activity. If this is the case, we must at least at times observe sufficiently high spectral densities to explain the highest observed temperatures, and this is indeed the case. Similarly, the lower than predicted observed temperatures for the very highest electric field spectral densities are consistent with a sporadic nature of the most enhanced wave activity. The ions have seldom experienced the heating for as long as is assumed by the theory at the time of observation. Some of the most intense heating is known to occur in a very limited region at the equatorward side of the cusp, sometimes called the "cusp heating wall" (Bouhram et al., 2003), possibly caused by a combination of waves and quasi-static electric fields (Lindstedt et al., 2010). This could provide $\mathrm{O}^{+}$ ions with energies of several $\mathrm{keV}$.

Finally, the models seems to overestimate the temperature for the lowest spectral densities. This agrees with the results of Waara et al. (2011) where it was found that the average wave activity could explain the average ion temperatures only for geocentric distances above $8 R_{\mathrm{E}}$. In more recent work, we have found that the fraction of the wave activity, which is efficient in heating the ions, must be lower at lower altitudes (Nilsson et al., 2012). This is likely the explanation for the overestimate of the temperature for low spectral density which mostly corresponds to low altitude. The initial conditions are not important at higher altitudes as the heating rate increases with altitude and the most recent heating thus dominates the result.

\section{Conclusions}

The $\mathrm{O}^{+}$temperature and wave intensity at the oxygen gyrofrequency at each measurement point for a high altitude cusp and mantle data set obtained with the Cluster spacecraft are clearly correlated. The trend of the correlation agrees with the predictions by both an asymptotic mean-particle theory and a test-particle approach. Also the temperature ratio is consistent with the predictions of the asymptotic meanparticle theory for our spectral slope $\alpha$. There is some spread in the correlation, in particular for some of the highest observed temperatures which are up to one order of magnitude higher than predicted by the model.

An explanation as to why high temperatures are sometimes observed for periods of comparatively low electric field spectral density is the relatively sporadic wave activity which makes it less likely to observe the actual heating. Waara et al. (2011) found that intense wave activity (above $\left.3\left(\mathrm{mV} \mathrm{m}^{-1}\right)^{2} \mathrm{~Hz}^{-1}\right)$ seldom occurred continuously for more than a few minutes. The majority of events lasted less than $5 \mathrm{~min}$ as observed by the spacecraft. A simple study indicates that enhanced perpendicular temperatures would be observed over many $R_{\mathrm{E}}$ after heating ceases. Waves with sufficient amplitudes to explain the highest observed temperatures are indeed observed, even though relatively infrequently, consistent with the expectations for sporadic heating. For the very highest wave activity observed, the models typically overestimate the temperature. This is further evidence for the sporadic nature of such high amplitude waves. 
Acknowledgements. M. Waara, R. Slapak and G. Stenberg were financed by the Swedish National Graduate School of Space Technology. H. Nilsson and M. André were supported by the Swedish National Space Board. We also thank the EFW, FGM and CIS instrument teams.

Topical Editor I. A. Daglis thanks K. Lynch and one anonymous referee for their help in evaluating this paper.

\section{References}

Balogh, A., Carr, C. M., Acuña, M. H., Dunlop, M. W., Beek, T. J., Brown, P., Fornacon, K.-H., Georgescu, E., Glassmeier, K.H., Harris, J., Musmann, G., Oddy, T., and Schwingenschuh, K.: The Cluster Magnetic Field Investigation: overview of in-flight performance and initial results, Ann. Geophys., 19, 1207-1217, doi:10.5194/angeo-19-1207-2001, 2001.

Bouhram, M., Malingre, M., Jasperse, J. R., Dubouloz, N., and Sauvaud, J.-A.: Modeling transverse heating and outflow of ionospheric ions from the dayside cusp/cleft. 2 Applications, Ann. Geophys., 21, 1773-1791, doi:10.5194/angeo-21-17732003, 2003.

Chang, T., Crew, G. B., Hershkowitz, N., Jasperse, J. R., and Retterer, J. M.: Transverse acceleration of oxygen ions by electromagnetic ion cyclotron resonance with broad band lefthand polarized waves, Geophys. Res. Lett., 13, 636-639, doi:10.1029/GL013i007p00636, 1986.

Escoubet, C. P., Fehringer, M., and Goldstein, M.: Introduction: The Cluster mission, Ann. Geophys., 19, 1197-1200, doi:10.5194/angeo-19-1197-2001, 2001.

Gustafsson, G., André, M., Carozzi, T., Eriksson, A. I., Fälthammar, C.-G., Grard, R., Holmgren, G., Holtet, J. A., Ivchenko, N., Karlsson, T., Khotyaintsev, Y., Klimov, S., Laakso, H., Lindqvist, P.-A., Lybekk, B., Marklund, G., Mozer, F., Mursula, K., Pedersen, A., Popielawska, B., Savin, S., Stasiewicz, K., Tanskanen, P., Vaivads, A., and Wahlund, J.-E.: First results of electric field and density observations by Cluster EFW based on initial months of operation, Ann. Geophys., 19, 1219-1240, doi:10.5194/angeo19-1219-2001, 2001.

Jasperse, J. R., Basu, B., Lund, E. J., and Bouhram, M.: Gyrotropic guiding-center fluid theory for the turbulent heating of magnetospheric ions in downward Birkeland current regions. II, Phys. Plasmas, 13, 112902, doi:10.1063/1.2364475, 2006.
Lennartsson, O. W., Collin, H. L., and Peterson, W. K.: Solar wind control of Earth's $\mathrm{H}^{+}$and $\mathrm{O}^{+}$outflow rates in the 15$\mathrm{eV}$ to 33-keV energy range, J. Geophys. Res., 109, A12212, doi:10.1029/2004JA010690, 2004.

Lindstedt, T., Khotyaintsev, Y. V., Vaivads, A., André, M., Nilsson, H., and Waara, M.: Oxygen energization by localized perpendicular electric fields at the cusp boundary, Geophys. Res. Lett., 37, L09103, doi:10.1029/2010GL043117, 2010.

Moore, T. E. and Horwitz, J. L.: Stellar ablation of planetary atmospheres, Rev. Geophys., 45, RG3002, doi:10.1029/2005RG000194, 2007.

Nilsson, H., Waara, M., Arvelius, S., Marghitu, O., Bouhram, M., Hobara, Y., Yamauchi, M., Lundin, R., Rème, H., Sauvaud, J.-A., Dandouras, I., Balogh, A., Kistler, L. M., Klecker, B., Carlson, C. W., Bavassano-Cattaneo, M. B., and Korth, A.: Characteristics of high altitude oxygen ion energization and outflow as observed by Cluster: a statistical study, Ann. Geophys., 24, 1099-1112, doi:10.5194/angeo-24-1099-2006, 2006.

Nilsson, H., Barghouthi, I. A., Slapak, R., and Eriksson, A. I.: Hot and cold ion outflow: observations and implications for numerical models, J. Geophys Res., submitted, 2012.

Retterer, J. M., Chang, T., Crew, G. B., Jasperse, J. R., and Winningham, J. D.: Monte Carlo modeling of ionospheric oxygen acceleration by cyclotron resonance with broad-band electromagnetic turbulence, Phys. Rev. Lett., 59, 148-151, doi:10.1103/PhysRevLett.59.148, 1987.

Slapak, R., Nilsson, H., Waara, M., André, M., Stenberg, G., and Barghouthi, I. A.: $\mathrm{O}^{+}$heating associated with strong wave activity in the high altitude cusp and mantle, Ann. Geophys., 29, 931-944, doi:10.5194/angeo-29-931-2011, 2011.

Waara, M., Nilsson, H., Stenberg, G., André, M., Gunell, H., and Rème, H.: Oxygen ion energization observed at high altitudes, Ann. Geophys., 28, 907-916, doi:10.5194/angeo-28-907-2010, 2010.

Waara, M., Slapak, R., Nilsson, H., Stenberg, G., André, M., and Barghouthi, I. A.: Statistical evidence for $\mathrm{O}^{+}$energization and outflow caused by wave-particle interaction in the high altitude cusp and mantle, Ann. Geophys., 29, 945-954, doi:10.5194/angeo-29-945-2011, 2011. 